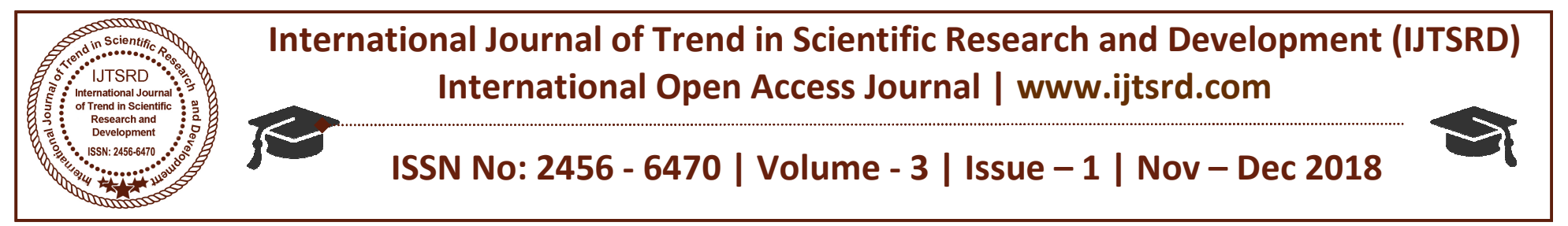

\title{
Performance Analysis of Different QAM Techniques Using Rician Fading Channel
}

\author{
Prerna Amlavad, Mukesh Ptidar \\ Department of Electronics and Communication, \\ Lakshmi Narain College of Technology (LNCT), Indore, Madhya Pradesh, India
}

\begin{abstract}
Wireless communications is one of the most active areas of technology development of our time. This development is being driven primarily by the transformation of what has been largely a medium for supporting voice telephony into a medium for supporting other services, such as the transmission of video, images, text, and data. In this paper QAM modulation techniques used for calculation of SNR with MIMO system using fading channel. This work presents, a simulation toll MATLAB R2013a to study the performance analysis of Bit Error rate (BER) V/S
\end{abstract} Signal to Noise ratio (SNR).

Key Words: BER, Fading Channel, MIMO System, QAM Modulation, SNR etc

\section{INTRODUCTION}

The wireless communication history, every generation of computers get advanced with new frequency bands, high data rates and non backwards compatible transmission technology. Here as we know $4 \mathrm{G}$ is a successor of $3 \mathrm{G}$, i.e. $4 \mathrm{G}$ provides internet broadband in computer devices and other mobile devices .Some of the other features you use now are days are parts of it like High definition Mobile TV, Video conferencing, video calling, accessing mobile internet, IP Telephony (Voice Over Internet Protocol [VIOP] it is the group of technologies which is used to deliver the voice communication through the internet protocol).

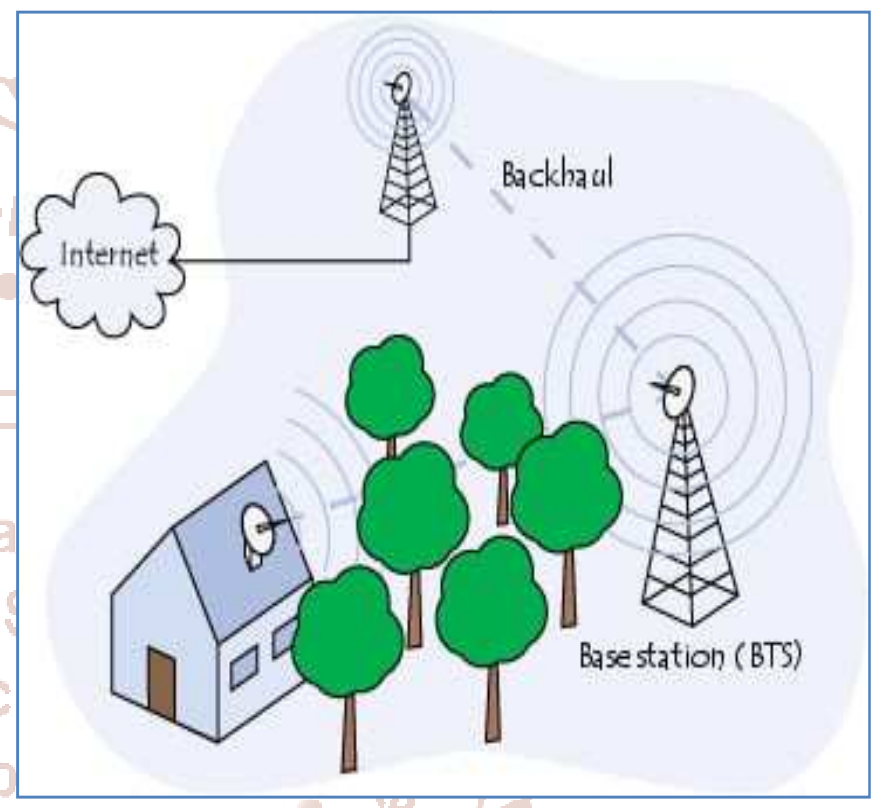

Fig.1: WiMAX System

The revisions of the IEEE 802.16 standard fall into two categories:

Fixed WiMAX, also called IEEE 802.16-2004, provides for a fixed-line connection with an antenna mounted on a rooftop, like a TV antenna. Fixed WiMAX operates in the $2.5 \mathrm{GHz}$ and 3.5 $\mathrm{GHz}$ frequency bands, which require a licence, as well as the licence-free $5.8 \mathrm{GHz}$ band.

> Mobile WiMAX, also called IEEE 802.16e, allows mobile client machines to be connected to the Internet. Mobile WiMAX opens the doors to mobile phone use over IP, and even high-speed mobile services.

\section{A. MIMO-OFDMA}

MIMO-OFDMA is a combination of downlink MIMO transmission and OFDM based multiple accesses. In multiple inputs multiple output (MIMO) communication System exploits the degrees of 
freedom introduced through multiple transmitted and received antenna to offer high spectral efficiency. In narrow band channels, when channels state information is available at the transmitter and instantaneous adaptation is possible, the capacity attaining distribution is found by using the wellknown water filling algorithm.

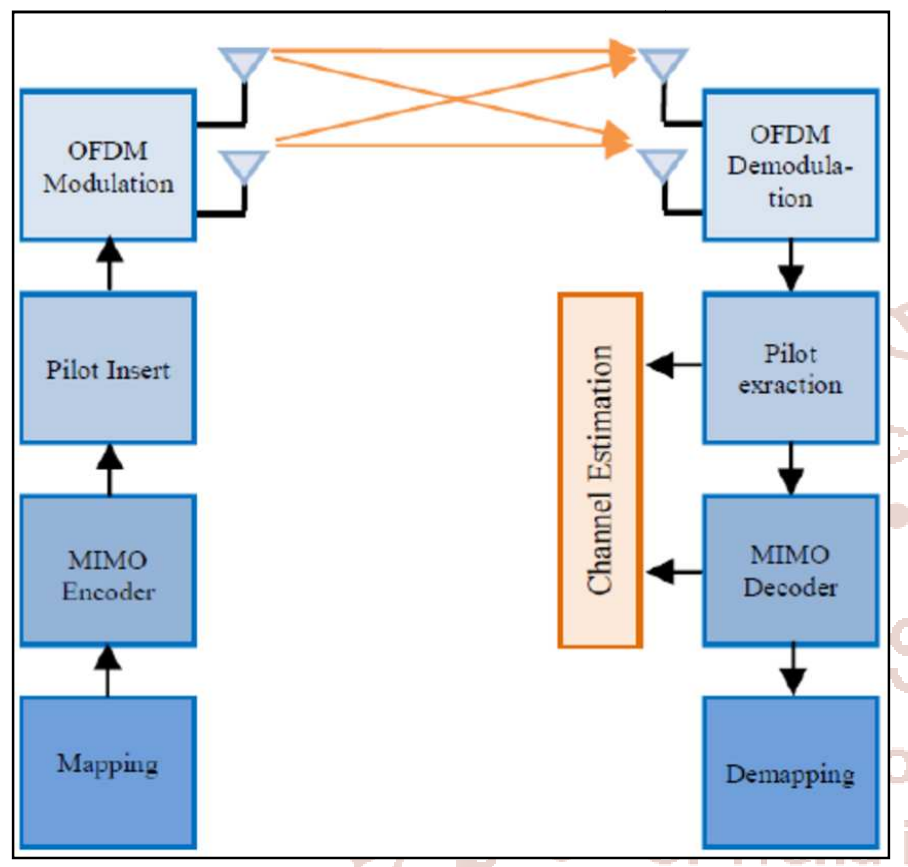

Fig. 2: MIMO-OFDMA System Model

\section{B. 4-QAM}

The 4-state Quadrature amplitude modulation (4QAM) is a QAM with $\mathrm{M}=2$ voltage levels or possible states for the signal. QAM transmits $\mathrm{k}$ bits of information during each symbol period, where $\mathrm{k}=\log _{2} \mathrm{M}=2$ bits, that is $\mathrm{M}=2^{\mathrm{k}}$. In this condition the symbol rate is half of the bit rate, producing a spectrally efficient transmission. The 16-state Quadrature amplitude modulation (16-QAM) is a QAM with $\mathrm{M}=16$ voltage levels or possible states for the signal, that is, four I value $\mathrm{Q}$ values. QAM transmits $\mathrm{k}$ bits of information during each symbol period, where $\mathrm{k}=\log _{2} \mathrm{M}=4$ bits, that is $\mathrm{M}=2^{\mathrm{k}}$, consisting of two bits for I and two bits for Q. And the symbol rate is one fourth of the bit rate, producing a very spectrally efficient transmission.

\section{C/ BLOCK DIAGRAM OF SIMULATION MODEL}

The simulation system is shown in figure below, the system will be developed the MIMO-OFDM system using MATLAB. The adaptive modulation controller is receiving signal number of transmission and number of receiver and SNR, in control OFDM transmission and OFDM receiver signal. The graphs shown in simulation \& result section of the paper clarify the process shown in the system model.

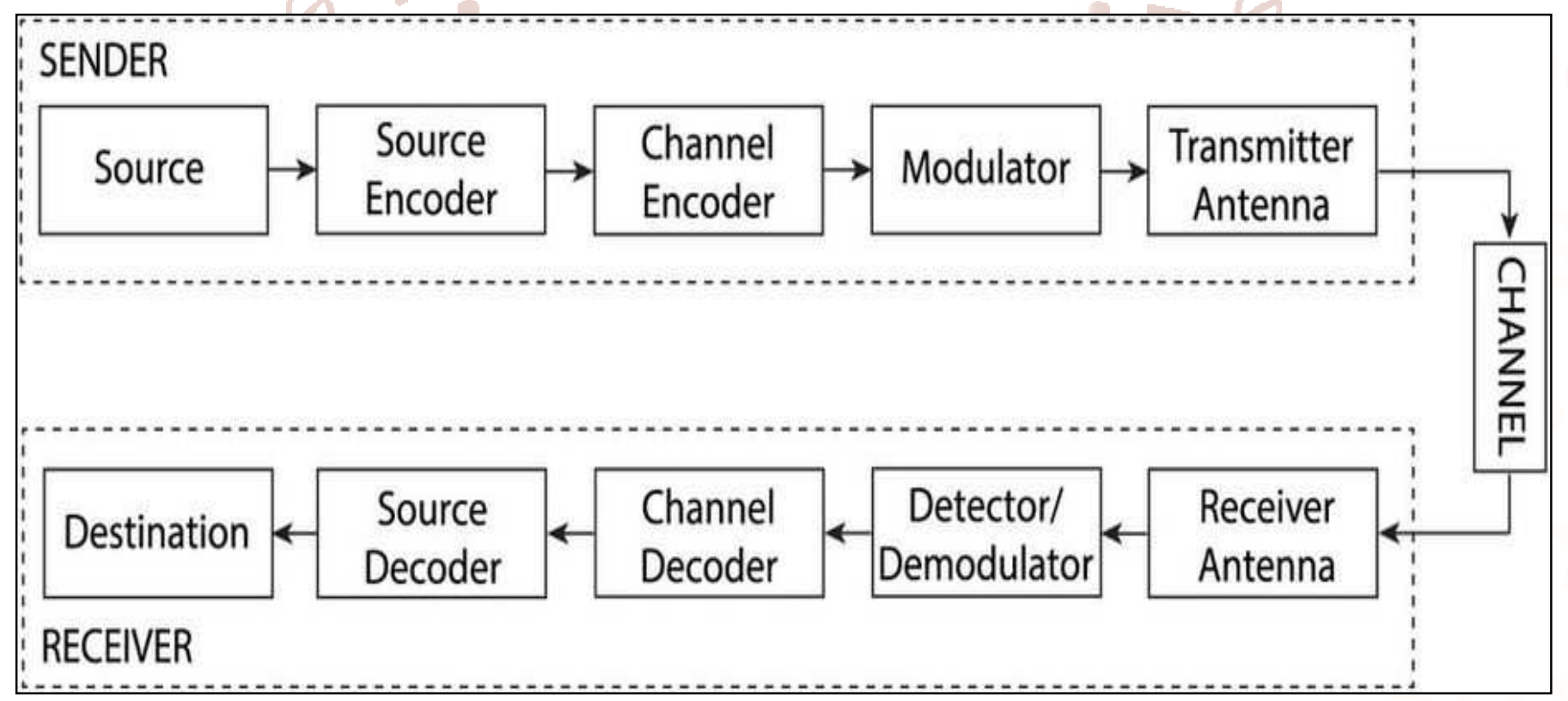

Fig. 3: System Block Diagram

\section{SIMULATION DIFFERENT QAM SYSTEM DIFFERENT CHANNEL}

In this paper work consider following input parameters are shown in table 1 . The different
FOR modulation scheme are consider like (4-QAM, 16WITH QAM and 64-QAM), with Rician Channel. The Carrier rate is $1 / 2$ with FFT size 256 consider. The project work done by using simulation tool MATLAB R2013a Used. 
International Journal of Trend in Scientific Research and Development (IJTSRD) ISSN: 2456-6470

Table: 1 The Simulation parameter

\begin{tabular}{|l|l|}
\hline \multicolumn{1}{|c|}{ Parameters } & \multicolumn{1}{c|}{ Value } \\
\hline Communication Channel & Fading \\
\hline Modulation Techniques & 4-QAM, 8-QAM and 16-QAM \\
\hline IFFT (Input port size) & 256 \\
\hline CC Code Rate & $1 / 2$ \\
\hline Radio Technology & OFDM \\
\hline Used Scheme & Alamouti \\
\hline Model & WiMAX 802.16e \\
\hline Calculation Parameters & BER V/s SNR \\
\hline Simulation-Used Tool/Software & Matlab (R2013a) \\
\hline
\end{tabular}

Rician fading best describes a situation where a dominant non-fading, line-of-sight (LOS), component is present in addition to a number of indirect multipath signals. The fading envelope of this model is described by Rician probability density function
(PDF). The performance of simulation results are fairly equals to theoretical results. The four color lines indicate the different QAM levels. The used communication channel Rician with QAM show in figure. 4.

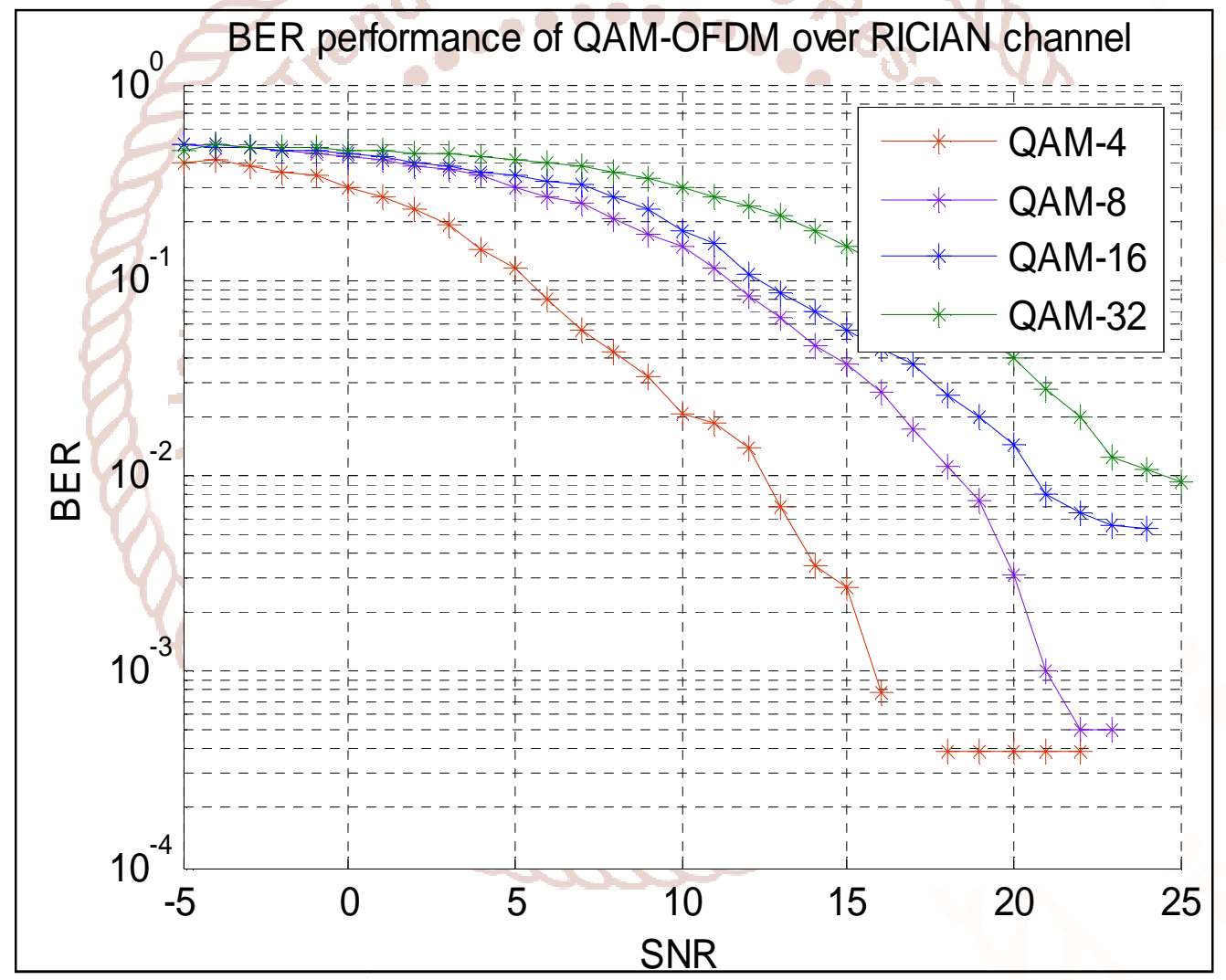

Fig: 4: BER performance of different QAM-OFDM over RICIAN

\section{RESULT ANALYSIS AND CONCLUSION}

In this performance, we have used the Alamouti scheme with communication Rician channel and different modulation techniques. The performance is displayed in figure 4 in terms of the BER verses SNR logarithmic plot. In the fig. 4 in this plot we analysis the 32-QAM, SNR is increased $4.1 \mathrm{~dB}$ on BER at $10^{-2}$ as compared to 16-QAM and Modulation Techniques.
References:

1. Mehdi Ahmadi, EhsanRohani, Pooya Monshizadeh Naeeni and Sied Mehdi Fakhraie, "Modeling and Performance Evaluation of IEEE 802.22 Physical Layer". 2nd International Conference on Future Computer and Communication (ICFCC)-2010, Wuhan, ISBN: 978-1-4244-5821-9, 21-24 May 2010, Vol. 3 V3-62 - V3-66, INSPEC Accession Number : 11538274 
2. Y. Li, J. H. Winters and N. R. Sollenberger, "MIMO-OFDM for Wireless Communications: Signal Detection with Enhanced Channel Estimation", IEEE Transaction on Communications, Vol. 50, Issue 9, pp 1471-1477, 2009.

3. S. Moghe and R. Upadhyay, "Comparison of SISO and MIMO Techniques in 802.11n Wireless Local Area Network", International Conference on Emerging Trends in Electronic and Photonic Devices \& Systems, pp 245-246, 2009

4. T.P. surekha, T. Ananthapadmanabha, C. Puttamadappa Members, IEEE, Modeling and Performance Analysis of QAM-OFDM System with AWGN Channel, C2011 IEEE

5. Sai Krishna Borra; Suman Krishna Chaparala, performance evolution of OFDM system with Rayleigh, racian and AWGN channels, Volume 3, Issue 3, March 2013.

6. Ashutosh Kumar Mishra, Rashmi Pandey, A Review on Modeling and Performance of QAMOFDM System with AWGN Channel, Volume 4, Issue 3, March 2014.
7. Kapil Narwal 1 , Yagvalkya Sharma 2, Performance Comparison of Turbo Codes with other Forward Error Correcting Codes, Jaipur National University, Jaipur, ISSN: 2277-1956.

8. Y. Huang, B. Pei, and H. Zhang, "System Performance Research and Analysis of MIMO OFDM Based on Space-Time Block Codes", International Conference on Intelligent Control and Information Processing, pp 414-417, 2010.

9. P. S. Mundra, T. L. Singal and R. Kapur, "The Choice of A Digital Modulation, Schemes in A Mobile Radio System", IEEE Vehicular Technology Conference, Issue 5, pp 1-4,1993.

10. G. Ganesan and P. Stoica. 2001. "Space-time block codes: a maximum SNR approach", IEEE Transactions on Information Theory, Vol. 47, Issue 4, May 2001, pp. 1650-1656.

11. A. Tarighat and A. H. Sayed, "MIMO OFDM receivers for systems with IQ imbalances," IEEE Transactions on Signal Processing, Vol. 53, Issue 9, pp. 3583-3596, September 2005. 